GS:1.0, DP:0.8, DAS28:0.9 and SJC:1.0). The average time required to score one patient was $140 \mathrm{~s}$.

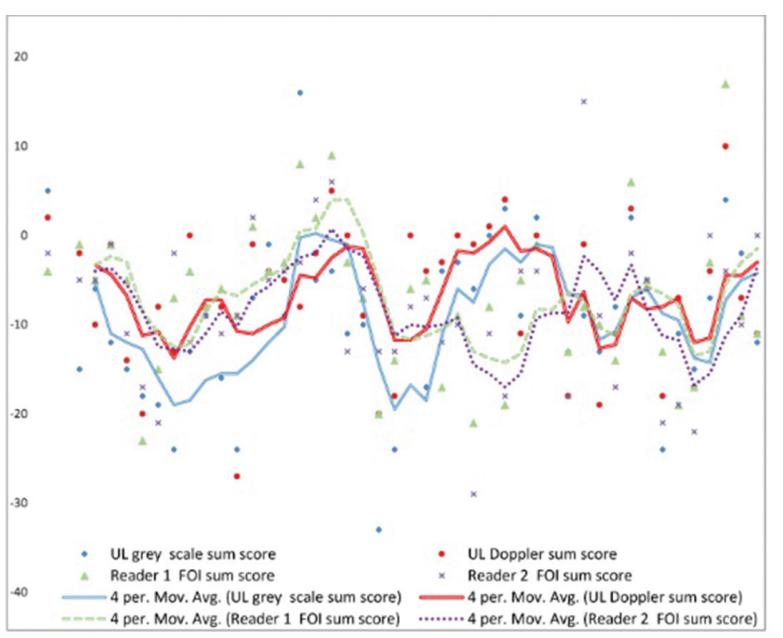

Abstract AB1202 - Figure 1. Scatterplot for FOI and US changes sum score and 4 patient avenges trendlines

Conclusions: This study shows a significant correlation and corresponding trendlines between FOI and US for change over time, and good responsiveness using the new FOI scoring system. FOI may therefore be used as an alternative to known imaging modalities for monitoring RA patients in clinical trials, and potentially in the daily clinical practice.

Disclosure of Interest: None declared

DOI: 10.1136/annrheumdis-2018-eular.3039

\section{AB1203 USE OF MAGNETIC RESONANCE IMAGING OF THE PELVIS TO DESCRIBE INFLAMMATORY CHANGES AT DIFFERENT ANATOMIC SITES IN THE PELVIS WHICH ARE POTENTIALLY SPECIFIC FINDINGS IN PATIENTS WITH POLYMYALGIA RHEUMATICA}

M. Fruth ${ }^{1}$, M. Franklin ${ }^{2}$, B. Buehring ${ }^{2}$, X. Baraliakos ${ }^{2}$, J. Braun ${ }^{2} .{ }^{1}$ Radiologie Herne; ${ }^{2}$ Rheumazentrum Ruhrgebiet, Herne, Germany

Background: Pelvic girdle pain is a common clinical symptom of patients with polymyalgia rheumatica (PMR). It also occurs in patients with rheumatoid arthritis (RA). The origin of this characteristic pain is not really clear, even though some imaging findings have been reported. However, this has neither changed pathophysiologic thinking nor clinical practice related to diagnosis.

Objectives: To describe pelvic structures in PMR patients in detail which show signs of inflammation by magnetic resonance imaging (MRI) in order to find a disease specific pattern.

Methods: In a retrospective study we used MRIs of patients who had presented with clinical symptoms suggestive of PMR in our centre between 2015 and 2017 . Only patients with complete MRI examinations, including contrast enhanced scans in coronal and transversal planes were included to be carefully examined by an experienced musculoskeletal radiologist (MF). After having first described all findings in much detail we developed a preliminary semiquantitative scoring system that assesses a total of 12 sites which appeared to be frequently involved. A total of 40 patients with pelvic girdle pain and complete data were identified from the hospital records. The median (25th/75th percentiles) age of the patients was $67(55 / 73)$ years, the median symptom duration was $13(6 / 22)$ weeks, $55 \%$ were female, the median C-reactive protein measured close to the day of the MRI examination was $1.9(0.7 / 4) \mathrm{mg} / \mathrm{dl}$, and the median erythrocyte sedimentation rate was 30 (17/43) after the 1st hour. Only 3 patients were rheumatoid factor positive, and 2 patients had anti-CCP antibodies. Ten patients had a diagnosis of RA $(25 \%)$ in addition to the leading PMR like symptom.

Results: The MRI signal of interest for PMR is a post-gadolinium signal in T1 weighted pelvic images. The most frequently involved anatomic sites were: the hamstring tendon and the M.gluteus medius and minimus tendon near the greater trochanter in all cases, which were found to be bilaterally involved as was the proximal M.rectus femoris in all cases, and the insertion of the adductor muscles, especially the M.adductor longus at the inferomedial pubic symphysis in $90 \%$ of cases. Other sites were also, but less frequently, involved. We think that the involvement of 4 sites including either the M.rectus femoris or the M.adductor longus is rather specific for PMR, see examplary images below. There was no major difference between patients with and without RA

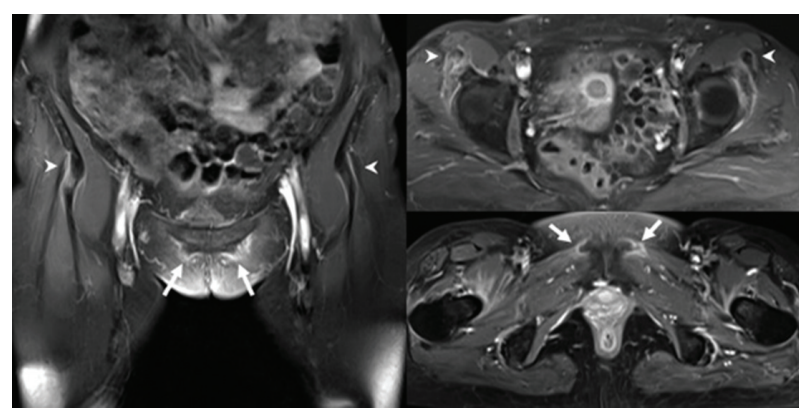

Abstract AB1203 - Figure 1

Conclusions: This study suggests that there may be a MRI pattern specific for PMR. The target structure of the characteristically inflamed anatomic site seems to be the paratenon which implies that the pattern observed in PMR differs from the enthesitis seen in patients with spondyloarthritis. Prospective randomised trials are needed to further test and prove the clinical usefulness of this approach. Disclosure of Interest: None declared DOI: 10.1136/annrheumdis-2018-eular.5225

\section{AB1204 QUANTITATIVE MRI AND DYNAMOMETERS CAN DISTINGUISH MYOSITIS FROM HEALTHY CONTROL MUSCLE}

M. Farrow ${ }^{1,2}$, J. Biglands ${ }^{2}$, S. Tanner ${ }^{2}$, M. Buch ${ }^{1,2}$, P. Emery ${ }^{1,2}$, A. Grainger ${ }^{2}$, A L. Tan ${ }^{1,2}{ }^{1}{ }^{1}$ Leeds Institute of Rheumatic and Musculoskeletal Medicine, University of Leeds; ${ }^{2}$ NIHR Leeds Biomedical Research Centre, Chapel Allerton Hospital, Leeds, UK

Background: Myositis is an autoimmune disease which can decrease quality of life and increase mortality. Clinical presentation includes muscle weakness, changes in muscle microstructure, myosteatosis, raised muscle enzymes and myalgia. Diagnosis is reliant on subjective clinical examinations, blood tests and invasive muscle biopsies. Quantitative MRI techniques such as diffusion tensor imaging (DTI) and fat fraction (FF) measurements offer non-invasive measurements, which could improve the understanding of muscle pathology and potentially inform diagnosis. DTI measures water diffusion within tissues which is sensitive to changes in muscle microstructure ${ }^{(1)}$. FF provides a quantitative measure of myosteatosis in muscles ${ }^{(2)}$. The use of these techniques could provide new imaging biomarkers in the diagnosis and management of myositis.

Objectives: To evaluate whether fat fraction, mean diffusivity and dynamomete measurements are sensitive enough to detect muscle differences in myositis patients compared to healthy controls.

Methods: 10 active myositis patients ( 6 female, mean age $55 \pm 18$ ) diagnosed according to the Bohan and Peter myositis criteria (mean CK 2,015 $\pm 10,787$ ) and 16 healthy controls ( 10 female, mean age $44 \pm 17$ ), were imaged using STEAMEPI diffusion and 2-point Dixon to obtain FF measurements. Myositis patients included 5 polymyositis, 3 dermatomyositis and 2 inclusion body myositis. Mean measurements of FF and mean diffusivity (MD) were obtained from regions drawn manually within the individual muscles of the quadriceps and hamstrings (image 1). No distinction was made between affected and unaffected muscles. In addition to MRI, all participants had knee extension and flexion power and torque measured on an isokinetic dynamometer and handgrip measurements on an isometric dynamometer. Differences were assessed using independent T-tests.

Results: FF and diffusion measurements were higher in myositis patients compared to healthy controls, whereas muscle power and torque were reduced (table 1). These results were consistent across both quadriceps and hamstrings.

Abstract AB1204 - Table 1

\begin{tabular}{|c|c|c|c|c|c|c|c|}
\hline & & \multicolumn{2}{|c|}{ Mean } & \multicolumn{2}{|c|}{$\begin{array}{c}\text { Mean difference } \\
(95 \% \mathrm{Cl})\end{array}$} & \multicolumn{2}{|c|}{$\frac{\text { Significance }}{\text { (p value) }}$} \\
\hline & & Hams & Quads & Hams & Quads & Hams & Quads \\
\hline \multirow[t]{2}{*}{$\begin{array}{l}\mathrm{FF} \\
(\%)\end{array}$} & Myositis & $\begin{array}{c}15.09 \\
(-9.21 \\
39.39)\end{array}$ & $\begin{array}{c}14.33 \\
(-16.95 \\
45.6)\end{array}$ & $\begin{array}{l}10.08 \\
(5.12- \\
15.05)\end{array}$ & $\begin{array}{c}11.2 \\
(5.79- \\
16.61)\end{array}$ & $<0.001$ & $<0.001$ \\
\hline & Healthy & $\begin{array}{c}5.0 \\
(-4.89, \\
14.82)\end{array}$ & $\begin{array}{c}3.13 \\
(-0.28 \\
6.54)\end{array}$ & & & & \\
\hline
\end{tabular}

